\title{
Inhibition of spinal bone formation in AS: 10 years after comparing adalimumab to OASIS
}

\author{
Désirée van der Heijde ${ }^{1 *}$ (D) and Robert Landewé2,3
}

\begin{abstract}
A decade has passed since the publication on the comparison of the effect of adalimumab with data from a historic cohort on the progression of structural damage in the spine of patients with ankylosing spondylitis (AS). No effect could be observed, and currently, there is still no definite proof that TNF inhibitors (TNFi) inhibit spinal structural damage. The findings of the publication are discussed within the context of the time at the publication and new developments.
\end{abstract}

Keywords: Ankylosing spondylitis, Structural damage, TNF inhibitor

In 2009, we published about the effects of 2-year treatment with the TNF inhibitor adalimumab on spinal radiographic progression in patients with ankylosing spondylitis (AS) [1]. The remarkable and somewhat unusual comparator group was formed by a historic cohort of patients with AS treated with NSAIDs (nonsteroidal anti-inflammatory drugs) and csDMARDs (conventional synthetic disease-modifying antirheumatic drugs): the OASIS (Outcome in AS International Study) cohort [2]. The scoring method was the mSASSS (modified Stoke AS Spinal Score), a method using conventional radiography [3]. There was no slowing of radiographic progression by adalimumab, neither in the full OASIS cohort nor in the subgroup of patients that retrospectively should have fulfilled the entry criteria for the adalimumab trials. Many people were disappointed by these "unexpected" results, even though similar results had been shown for etanercept and infliximab [4, 5]. Still, people kept hope that a different monoclonal antibody against TNF should prove inhibition of bone proliferation, likely in view of inhibitory effects of these drugs in RA (rheumatoid arthritis) and PsA (Psoriatic Arthritis). But, as shown in the paper, the observed progression in the three groups of patients treated with a TNFi and the

\footnotetext{
* Correspondence: mail@dvanderheijde.nl

'Department of Rheumatology, Leiden University Medical Center, P.O. Box 9600, 2300 RC Leiden, The Netherlands

Full list of author information is available at the end of the article
}

three independent reads of the same OASIS films showed very similar mean progression rates of 0.8 to 1.0 (SD 2.6-3.3) units. Obviously, the reading by the mSASSS method was very robust, as each assessment was performed by different reader pairs and yielded consistent 2-year rates of progression in four cohorts of patients (i.e., three trials and OASIS).

The different reactions to these disappointing results could be distinguished in two opposing opinions: (1) "there must be an effect, so if you do not see it there will be good reasons to explain this" and (2) "the pathophysiology of the underlying bone processes in RA (bone-destruction) and AS (bone formation) differ diametrically, which explains the lack of inhibitory effect on spinal bone formation." Some hypotheses supporting the first view ("you missed the effect") included differences in severity (disease activity and prognostic factors) between the patients treated with adalimumab and those from OASIS, a too short period of depression of inflammation in a 2-year trial, a too late start in the disease to prevent reparative processes leading to bone formation, and a too insensitive outcome measure (mSASSS). The opponents of the "expected effect" hypothesis argued that bone formation often develops at sites without inflammation, that the Wnt signaling pathway is more involved in bone formation than the TNF pathway, and even that extra bone formation could occur when TNF is inhibited $[6,7]$. 
What have we learned in the subsequent decade? A very important finding was the formal proof of a longitudinal relationship between (increase in) disease activity (assessed as ASDAS (AS Disease Activity Score) and subsequent (increase in) mSASSS [8]. Another important finding was that the presence of inflammation at a vertebral corner, as seen on MRI, increases the probability of the formation of a syndesmophyte at that site on a radiograph 2 years later. It was made likely that this happens at those corners in which inflammation disappears and is replaced by fatty infiltration, but not at corners with persistent inflammation [9]. Still, the majority of syndesmophytes develop at corners without (observed) inflammation on MRI. The robust relationship between disease activity and MRI inflammation on one side and structural damage on the other side adds to the likelihood that a TNFi-induced suppression of disease activity (either measured clinically as a decrease of ASDAS or on sequential spinal MRIs) may also lead to inhibition of spinal bone formation.

Several cohort data have been published addressing the effects of TNFi [10-12]. However, cohort data suffer from various issues (such as confounding by indication, selection of patients with available radiographs, radiographs not taken in relation to start of $\mathrm{TNFi}$, different intervals between radiographs, insufficient information about other treatments) [13]. Sophisticated statistical modeling aims to overcome these issues, but many assumptions have to be made and statistics do not adjust for unknown information. Notwithstanding these issues, several cohorts suggest an inhibitory effect of TNFi on radiographic progression, with various levels of persuasion. These effects are especially seen with longer follow-up ( 4 instead of 2 years), when disease activity is reduced or when TNFi is combined with NSAIDs [10, 11].

Despite all technical progress in the field of imaging, conventional radiography of the spine and the mSASSS as a scoring method have survived the "ravages of time" [13]. Still, they have important disadvantages such as insufficient resolution, inclusion of only half the spine (because of overprojection by pulmonary tissue), and poor sensitivity to change; a follow-up of at least 2 years is needed to demonstrate sufficient progression. Recently, a new scoring method exploiting images obtained by low-dose CT scans was presented $[14,15]$. The resolution of CT has always been superior to that of conventional radiography, but widespread application (e.g., in trials) was limited by prohibitory radiation dose levels. Software adaptations allowed the acquisition of proper quality CT images using far lower doses of radiation, and CT has thus become a feasible alternative. Most importantly, low-dose CT includes the whole spine and has in the meantime already proven far higher sensitivity to change. These developments may allow studies with lower numbers of patients and a shorter follow-up but still sufficient statistical power to demonstrate a difference in bone formation if it really exists.

Comparisons of contemporary trial populations with historical cohorts without b(biological)DMARD use such as OASIS have become less attractive since contemporary trials now likely include less severe patients than in the early years of TNFi trials. Having said that, since new treatments for AS, such as IL17i, have become available recently, it will now be possible and ethically justifiable to perform a head-to-head trial with two active treatments (i.e., TNFi vs. IL17i) for a period of 2 years. Such a trial may provide an answer to the question if bDMARDs inhibit bone proliferation in AS, but only if one of both treatments has a larger impact on structural damage progression than the other treatment. If both classes of bDMARDs reduce progression of bone formation equally well, this matter will remain concealed, but with the advent of additional new treatments, the likelihood of a differential effect on syndesmophyte formation will increase. It may still take another decade to get the final answer to the question if there is really a treatment for AS that reduces spinal bone proliferation and bamboo spine formation.

\section{Abbreviations \\ AS: Ankylosing spondylitis; ASDAS: AS Disease Activity Score; csDMARDs: Conventional synthetic disease-modifying antirheumatic drugs; mSASSS: Modified Stoke AS Spinal Score; NSAIDs: Nonsteroidal anti- inflammatory drugs; OASIS: Outcome in AS International Study; PsA: Psoriatic arthritis; RA: Rheumatoid arthritis \\ Acknowledgements \\ Not applicable}

Authors' contributions

Both authors drafted the text and approved the final version for publication.

Funding

No funding

Availability of data and materials

Not applicable

Ethics approval and consent to participate

Not applicable

Consent for publication

Not applicable

\section{Competing interests}

Désirée van der Heijde has received consulting fees from AbbVie, Amgen, Astellas, AstraZeneca, BMS, Boehringer Ingelheim, Celgene, Cyxone, Daiichi, Eisai, Eli-Lilly, Galapagos, Gilead, Glaxo-Smith-Kline, Janssen, Merck, Novartis, Pfizer, Regeneron, Roche, Sanofi, Takeda, and UCB Pharma and is Director of Imaging Rheumatology BV. Robert Landewé has received Consulting fees and/or research grants from AbbVie, Ablynx, Amgen, AstraZeneca, BristolMyers Squibb, Centocor, Galapagos, GlaxoSmithKline, Janssen, Eli Lilly, Merck, Novartis, Pfizer, Roche, Schering, and UCB Pharma and is Director of

Rheumatology Consultancy BV.

\section{Author details}

${ }^{1}$ Department of Rheumatology, Leiden University Medical Center, P.O. Box 9600, 2300 RC Leiden, The Netherlands. ${ }^{2}$ Amsterdam University Medical 
Center (ARC), Amsterdam, The Netherlands. ' 3 Zuyderland Medical Center, Heerlen, The Netherlands.

Received: 15 October 2019 Accepted: 30 October 2019

Published online: 06 November 2019

\section{References}

1. van der Heijde D, Salonen D, Weissman BN, Landewé R, Maksymowych WP, Kupper $\mathrm{H}$, et al. Assessment of radiographic progression in the spines of patients with ankylosing spondylitis treated with adalimumab for up to 2 years. Arthritis Res Ther. 2009;11:R127.

2. van der Heijde D, Landewé R, van der Linden S. How should treatment effect on spinal radiographic progression in patients with ankylosing spondylitis be measured? Arthritis Rheum. 2005;52:1979-85.

3. Creemers MC, Franssen MJ, van't Hof MA, Gribnau FW, van de Putte LB, van Riel PL. Assessment of outcome in ankylosing spondylitis: an extended radiographic scoring system. Ann Rheum Dis. 2005:64:127-9.

4. van der Heijde D, Landewé R, Einstein S, Ory P, Vosse D, Ni L, et al. Radiographic progression of ankylosing spondylitis after up to two years of treatment with etanercept. Arthritis Rheum. 2008;58:1324-31.

5. van der Heijde D, Landewé $R$, Baraliakos X, Houben H, van Tubergen A, Williamson P et al. Ankylosing Spondylitis Study for the Evaluation of Recombinant Infliximab Therapy Study Group. Radiographic findings following two years of infliximab therapy in patients with ankylosing spondylitis. Arthritis Rheum 2008:58:3063-3070.

6. van der Heijde D, Machado P, Braun J, Hermann K-GA, Baraliakos X, Hsu B, et al. MRI inflammation at the vertebral unit only marginally predicts new syndesmophyte formation: a multilevel analysis in patients with ankylosing spondylitis. Ann Rheum Dis. 2012;71:369-73.

7. Diarra D, Solina M, Polzer K, Zwerina J, Ominsky MS, Dwyer D, et al. Dickkopf-1 is a master regulator of joint remodeling. Nat Med. 2007;13:156-63.

8. Ramiro S, van der Heijde D, van Tubergen A, Stolwijk C, Dougados M, van den Bosch F, et al. Higher disease activity leads to more structural damage in the spine in ankylosing spondylitis: 12-year longitudinal data from the OASIS cohort. Ann Rheum Dis. 2014;73:1455-61.

9. Machado PM, Baraliakos X, van der Heijde D, Braun J, Landewé R. MRI vertebral corner inflammation followed by fat deposition is the strongest contributor to the development of new bone at the same vertebral corner: a multilevel longitudinal analysis in patients with ankylosing spondylitis. Ann Rheum Dis. 2016;75:1486-93.

10. Haroon N, Inman RD, Learch TJ, Weisman MH, Lee M, Rahbar MH, et al. The impact of tumor necrosis factor a inhibitors on radiographic progression in ankylosing spondylitis. Arthritis Rheum. 2013;65:2645-54.

11. Molnar C, Scherer A, Baraliakos X, de Hooge M, Micheroli R, Exer P, et al. TNF blockers inhibit spinal radiographic progression in ankylosing spondylitis by reducing disease activity: results from the Swiss Clinical Quality Management cohort. Ann Rheum Dis. 2018;77:63-9.

12. Machado P. Anti-tumor necrosis factor and new bone formation in ankylosing spondylitis: the controversy continues. Arthritis Rheum. 2013; 65:2537-40.

13. van der Heijde D, Braun J, Deodhar A, Baraliakos X, Landewé R, Richards HB, et al. Modified stoke ankylosing spondylitis spinal score as an outcome measure to assess the impact of treatment on structural progression in ankylosing spondylitis. Rheumatology (Oxford). 2019;58:388-400.

14. de Bruin F, de Koning A, van den Berg R, Baraliakos X, Braun J, Ramiro $S$, et al. Development of the CT Syndesmophyte Score (CTSS) in patients with ankylosing spondylitis: data from the SIAS cohort. Ann Rheum Dis. 2018;77:371-7.

15. de Koning A, de Bruin F, van den Berg R, Ramiro S, Baraliakos X, Braun J, et al. Low-dose $C T$ detects more progression of bone formation in comparison to conventional radiography in patients with ankylosing spondylitis: results from the SIAS cohort. Ann Rheum Dis. 2018;77:293-9.

\section{Publisher's Note}

Springer Nature remains neutral with regard to jurisdictional claims in published maps and institutional affiliations. 